Entorno Geográfico | $\mathbf{N}^{\circ} 23$ | enero-julio 2022 | pp. 1-29 | ISSN (en línea): 2382-3518 | e20111712 | Universidad del Valle

DOI: 10.25100/eg.v0i23.11712

Espacios y Territorios

\title{
Análisis de recorridos vivienda-trabajo productivo realizados por mujeres con distinta carga reproductiva en la zona metropolitana de Monterrey, México
}

\section{Analysis of housing/work commuting made by women who observe different reproductive workloads in the metropolitan area of Monterrey, Mexico}

\author{
Malinalli Hernández-Reyes \\ Instituto Tecnológico y de Estudios Superiores de Monterrey (ITESM), Monterrey, México. \\ malinallih@yahoo.com | 0000-0003-4140-9673
}

\begin{abstract}
Para citar este artículo: Hernández-Reyes, M. (2022). Análisis de recorridos vivienda-trabajo productivo realizados por mujeres con distinta carga reproductiva en la zona metropolitana de Monterrey, México. Entorno Geográfico, (23), e20111712. https://doi.org/10.25100/eg.v0i23.11712
\end{abstract}

\section{Resumen}

El objetivo de este trabajo es determinar la existencia de diferencias de longitud entre trayectos vivienda-trabajo productivo (viv-TP) efectuados por mujeres que observan distintas cargas de trabajo reproductivo (CTR). Para ello, se realizan un análisis estadístico y uno comparativo de corte geográfico a datos brindados por el Instituto Nacional de Estadística y Geografía (INEGI), correspondientes a la zona metropolitana de Monterrey (ZMM), México. Es interés también conocer si tales indicadores se relacionan con el sector económico de ocupación y el nivel de ingresos de las mujeres estudiadas. Los hallazgos hablan de una conexión entre las CTR y la localización, en función de la vivienda, del trabajo remunerado. Apuntan que, a mayor CTR, menor es la distancia viv-TP, cuestión que afecta tanto a la ocupación económica como a su nivel de ingresos: en términos relativos, más mujeres se ubican en trabajos demandantes de especialización profesional y de ingreso superior en los trayectos más largos; en cambio, hay más mujeres en actividades menos especializadas y menos remuneradas en los trayectos cortos.

Palabras clave: geografía de género, zona metropolitana de Monterrey (ZMM), trabajo reproductivo, ciudad funcionalmente dividida, segregación espacial.

\section{Abstract \\ This paper aims to identify the differences between housing/work commuting distances (hou/W) made by women who observe different reproductive workloads (RWL). Statistical data analysis and geographic comparison were performed to data from the National Institute of Statistic and Geography (INEGI, by its acronym in Spanish) for the metropolitan area of Monterrey (MAM), Mexico. It is also interesting to know whether these indicators are related


to the women's economic sector and income levels. The results suggest a trade-off between the RWL and the location of paid work. The higher the RWL, the shorter the commute from home to productive work, which affects both the economic occupation and their income level. In relative terms, there are more women in jobs with longer commutes which requires professional specialization with higher incomes. On the other hand, there are more women in less specialized activities with lower incomes on short commutes.

Keywords: gender geography, metropolitan area of Monterrey (MAM), reproductive work, functionally fragmented city, spatial segregation.

Recibido: 23 de abril de 2021

Aceptado: 22 de julio de 2021

\section{Introducción}

La construcción social de la ciudad tiene repercusiones sobre la vida de las personas, entre las que se observan las conectadas a la actividad económica de las mujeres, de particular interés en esta investigación.

La exclusión y segregación de la mujer en el ámbito económico mediante su atadura a la actividad doméstica y de cuidado es afianzada, entre otras cosas, por su discriminación en el diseño y construcción del espacio urbano, el cual proyecta "la propia división sexual del trabajo" (Col·lectiu Punt 6, 2019: 100). Darke (1998) lo explica de manera muy clara al apuntar que a la hora de planificar o intervenir las ciudades se asume que la mujer cumple únicamente el papel de administradora del hogar, sin advertir labor retribuida alguna, lo que repercute en la separación física de zonas residenciales y centros de trabajo que problematiza, aún más, su entrada al mundo laboral debido a los traslados entre unas y otras y al tiempo que ello conlleva.

En primera instancia, este hecho ha favorecido a que las mujeres se mantengan sumergidas en las actividades domésticas y de cuidado, lejos de las económicas (Darke, 1998), y a que participen en tareas productivas distintas a las desempeñadas por los varones, esto es, de manera segregada, en una segunda instancia (Hanson \& Pratt, 1995). Y mantenerse sumergidas en las actividades domésticas y de cuidado supone su segregación espacial hacia el ámbito privado, mediante su exclusión del espacio público de la ciudad (Segovia \& Rico, 2017).

Ya que el vínculo de hombres y mujeres con su vivienda es distinto (en unos más débil que en otras) dado el papel que se les ha asignado según su sexo -el rol femenino es el de madre y esposa, mientras que el masculino es el de proveedor del hogar (Massey, 1994; Mcdowell, 2000)- la ubicación de su lugar de trabajo retribuido dentro de la ciudad será también distinta. El trabajo de ellos tenderá a estar más lejos de la vivienda que el de ellas, y el tipo de ocupación será diferente a razón de la distribución heterogénea de la oferta laboral en un contexto urbano zonificado o dividido en funciones - residencial, industrial/financiera, 
comercial/de esparcimiento y de comunicación, basadas en las cuatro necesidades primordiales de habitar, trabajar, descansar y circular (García, 2016).

Para las mujeres, a diferencia de los varones, es importante que el desempeño de la labor remunerada (trabajo productivo) le permita no descuidar 'su' responsabilidad doméstica y de cuidado (trabajo reproductivo), motivo por el que tiende a ubicarse en trabajos próximos a su hogar y/o con flexibilidad de horario. Así, sus posibilidades laborales se reducen a las ofertadas en las cercanías de su vivienda, cuyos giros resultan ser distintos a los de las zonas industriales y distritos financieros, así como los de menor ingreso (Hanson \& Pratt, 1991, 1995; Madden, 1981; Næss, 2006).

Con base en lo expuesto, esta investigación pretende desvelar si la carga de trabajo reproductivo (CTR) incide de alguna manera en la ubicación geográfica del trabajo remunerado femenino. Su principal interés se centra en conocer si existen diferencias en la longitud de los trayectos vivienda-trabajo productivo (viv-TP) efectuados por mujeres que observan distintos niveles de trabajo doméstico y de cuidado, y si dicha longitud guarda algún tipo de relación con el sector económico de ocupación y su nivel de ingresos. Se parte de la hipótesis de que, a mayor CTR, menor es la distancia viv-TP, lo que se traduce en segregación espacial a nivel urbano, segregación ocupacional, y segregación en la percepción económica.

\section{Materiales y método}

Se lleva a cabo entonces un estudio de corte cuantitativo impulsado, en una primera fase, por un análisis estadístico de datos, y concretado en una segunda fase por medio de un análisis comparativo de los trayectos, en función de la intensidad o carga del trabajo doméstico y de cuidado de las mujeres que los integran, lo que permite la utilización del dato espacial en su 'versión geográfica'.

Los datos elegidos para llevar a cabo tales análisis, correspondientes a las variables enlistadas líneas abajo, provienen de la Muestra censal del Censo de Población y Vivienda 2010 (INEGI, 2010c), ya que es la fuente más completa, conveniente y reciente a la fecha. Estos se refieren a la zona metropolitana de Monterrey (ZMM), Nuevo León, México, y se encuentran desagregados a nivel municipal. Dicha muestra es estadísticamente representativa del total de la población mexicana y es calculada por el Instituto Nacional de Estadística y Geografía (INEGI, 2010b).

Se identifica como sujeto de estudio a las mujeres residentes de cualquiera de las trece municipalidades que en 2010 conformaban la ZMM, en el desempeño de un trabajo remunerado. En términos del INEGI, la mujer ocupada (MO), lo que resulta en un universo de 22408 sujetas.

Debido a la escala municipal de los datos, se establecen como unidades de análisis a cada uno de los trece municipios de la ZMM. 
La fase estadística se integra por una regresión por mínimos cuadrados ordinarios, $M C O$ (Wooldridge, 2015) (en hoja de cálculo Microsoft Excel, Add-ins Analysis Toolpak), y una regresión multi-logística (Greene, 2008) (en el software Stata, de StataCorp). Esta se ocupa de indagar acerca del nivel de relación entre la separación viv-TP y las variables de situación conyugal, número de hijos, nivel académico, nivel de ingreso y sector económico de ocupación.

Para la segunda fase, la espacial, son de interés los indicadores trayectos viv-TP y CTR experimentada por las MO. En cuanto a los viajes viv-TP, la variable de interés es la de longitud (para su cálculo y trazo se utilizó el sistema de información geográfica libre y de código abierto QGIS). En lo concerniente al segundo indicador, los hallazgos de la bibliografía consultada (Belaunzarán, 2017; Fagnani \& Chauviré, 1989; Hanson \& Pratt, 1995; Mojica, 2014; Orozco, 2017; Salazar, 1999), coinciden en que la CTR experimentada por las mujeres está fuertemente conectada a las variables demográficas de edad, estado conyugal, número de hijos, edad del hijo más pequeño, parentesco con el jefe o jefa del hogar y nivel académico, razón por la que es a través de ellas que se estima el valor de este indicador. Se establecen varias condiciones para cada una de estas variables, en atención tanto a lo revisado a nivel metodológico como a la disponibilidad de los datos, con el fin de que su análisis sea más accesible: 12-29 años, 30-59 años y 60 años y más (edad); en pareja y sin pareja (estado conyugal); un hijo, dos hijos y tres o más hijos (número de hijos); 0-9 años, 10-17 años y 18 años y más (edad del hijo más pequeño); esposa, hija y jefa (parentesco con el jefe o jefa del hogar); por último, sin prepa, con prepa o carrera técnica y con licenciatura o más (nivel académico). El objeto de esta etapa es comparar las distancias vivTP frente a los niveles de CTR.

En ambas fases se considera que los recorridos viv-TP corresponden a las distancias entre los centroides de las manchas urbanas de los municipios de residencia y del trabajo remunerado de la MO, pues el nivel de desagregación de los datos es el municipal. Esto debido a que no se cuenta con la información suficiente para determinar con mayor exactitud la localización de la vivienda ni del trabajo remunerado de las mujeres del estudio.

Con el propósito de tener una mayor certeza sobre las CTR de las mujeres, y ante la posibilidad de un análisis más detallado a medida que se acota el universo de análisis, se lleva a cabo una revisión de la composición familiar de los hogares a los que pertenece cada mujer, dentro de la segunda etapa del estudio.

En este punto, el hogar de la mujer estudiada se convierte en la unidad de análisis, cuya conformación puede determinarse mediante la variable identificación de vivienda, ID_VIV (INEGI, 2010c), asignada a cada individuo registrado en la base de datos consultada.

Se ha visto que la participación de las personas en la labor reproductiva depende grandemente de su sexo y de su posición o parentesco con relación al jefe o jefa del hogar, así como de la etapa del ciclo de vida familiar del hogar al que pertenecen -determinada por la presencia y edad de los hijos-, y del traslape de esos ciclos -definido por la existencia de integrantes con 
parentesco ascendente (padres o suegros) y/o descendente (nietos o nueras y yernos)(Orozco, 2017).

Orozco (2017) realiza un análisis a detalle del trabajo doméstico y de cuidado, y establece para ello la siguiente tipología de arreglos familiares: 1) no familiares; 2) biparental sin hijos; 3) biparental con al menos un hijo menor de 15 años; 4) biparental con hijos de 15 años y más; 5) monoparental con al menos un hijo menor de 15 años; 6) monoparental con hijos de 15 años y más; 7) extenso y compuesto con parentesco ascendente; y 8) extenso y compuesto con parentesco descendente. La autora atribuye una demanda o carga de trabajo reproductivo a cada arreglo familiar, basada en las horas promedio semanales que se le dedican a esa actividad, y observa que esta se conecta con el tamaño y el ciclo de vida familiares: los arreglos extensos y compuestos ascendentes y descendentes, además de los biparentales con al menos un hijo menor de 15 años, son los que ostentan las mayores cargas. Asimismo, demuestra que es el género femenino el que invierte más tiempo en la labor doméstica de todos los arreglos familiares. De igual forma, calcula la distribución de esta carga desagregando la posición o parentesco de los miembros de cada uno de esos arreglos.

La Tabla 1 se basa en la información generada por la investigadora citada y es utilizada para calcular, con una mayor precisión, la CTR de las mujeres de interés. En ella se le asigna a cada mujer una CTR diferente, reducida o considerable, de acuerdo a la conjunción parentesco/arreglo familiar del hogar al que pertenece ${ }^{1}$.

Tabla 1. Arreglos familiares y sus cargas de trabajo doméstico y de cuidado (CTR).

\begin{tabular}{|c|c|c|}
\hline Parentesco & Arreglo familiar & CTR \\
\hline N/A & No familiar (vive sola) & reducida \\
\hline Esposa & Biparental sin hijos & considerable \\
\hline Esposa & Biparental con hijos menores de 18 años & considerable \\
\hline Esposa & Biparental con hijos de 18 años y más & considerable \\
\hline Hija & Biparental con hijos & reducida \\
\hline Hija & Monoparental con hijos & reducida \\
\hline Jefa & Monoparental con hijos menores de 18 años & considerable \\
\hline Jefa & Monoparental con hijos de 18 años y más & considerable \\
\hline Jefa & Biparental con hijos & reducida \\
\hline Esposa/Hija/Jefa/Otro & Extenso A* & reducida \\
\hline Esposa/Hija/Jefa/Otro & Extenso B* & Considerable \\
\hline
\end{tabular}

Fuente: Elaboración propia con base en los datos del Cuadro 6 elaborado por Orozco (2017, p. 298).

${ }^{1}$ Los arreglos familiares y las CTR se basan en lo establecido por Orozco (2017), aunque los primeros se adecuan a las composiciones familiares halladas en la fuente de información. 
* El arreglo familiar del tipo Extenso implica la presencia de integrantes con parentesco ascendente y/o descendente. No obstante la aparente similitud entre el $A$ y el $B$, las CTR son distintas para cada uno, pues estas se establecen a partir del resto de los integrantes del hogar, caso por caso, en función de sus edades, sexo, parentesco, condición de ocupación y horas trabajadas a la semana.

\section{Sobre el contexto de estudio}

\section{La zona metropolitana de Monterrey (ZMM)}

La ciudad de Monterrey, fundada en 1596, experimentó un proceso de metropolización ${ }^{2}$ en función de sus modelos económicos, los cuales la llevaron de ser un pequeño asentamiento dedicado a la actividad mercantil, a convertirse en la gran metrópoli de la actualidad, condición detonada por la industrialización y consolidada por la terciarización ${ }^{3}$ característica de la época global (Aparicio Moreno et al., 2011; Contreras, 2007; Garza, 2010).

La actual ZMM se localiza al noreste de la República Mexicana, en el estado fronterizo de Nuevo León (Figura 1). La Secretaría de Desarrollo Social, el Consejo Nacional de Población y el Instituto Nacional de Estadística y Geografía (SEDESOL et al., 2012) señalan que, para 2010, eran trece los municipios que la integraban: Apodaca, Cadereyta Jiménez, Carmen, García, San Pedro Garza García, General Escobedo, Guadalupe, Juárez, Monterrey, Salinas Victoria, San Nicolás de los Garza, Santa Catarina y Santiago. Ese mismo año, ocupaba el tercer lugar a nivel nacional en concentración de población, después de las ZM del Valle de México y de Guadalajara; entonces contaba con 4'106,054 habitantes, el 88\% del total de la población estatal (con datos de INEGI, 2011; SEDESOL et al., 2012). Su superficie era de $6,794 \mathrm{~km}^{2}$, y presentaba una densidad media urbana (DMU) de 109.1 habitantes/hectárea. Las mujeres representaban el $50.14 \%$ y los hombres el $49.86 \%$ sobre el total de los habitantes a nivel estatal (INEGI, 2011).

\section{Zonificación o división funcional de la ZMM}

Ya que la metropolización de la región de Monterrey fue impulsada grandemente por su actividad económica, y esta a su vez guarda un fuerte vínculo con la especialización o perfil vocacional municipal, se realiza una revisión del sector económico al que pertenece la totalidad de las unidades económicas ${ }^{4}$ (UE) de los municipios metropolitanos, principalmente con dos objetivos. El primero, disponer de un panorama general actualizado a 2010 del comportamiento de la economía que contextualice la actividad remunerada

\footnotetext{
${ }^{2}$ Las etapas de metropolitanismo se caracterizan por el desplazamiento territorial tanto de población como de actividades económicas, observado del centro a la periferia (Sobrino, 2003).

3 La Revolución Terciaria (terciarización, servicialización) es un fenómeno vinculado a la creciente importancia del sector terciario en la economía que impacta, eventualmente, en el sector secundario, en el que se observa una disminución de sus niveles de empleabilidad y de producción (Contreras, 2007; Vásquez, 2009).

${ }^{4}$ Se entiende por unidad económica a la "unidad de observación sobre la cual se solicita y se publica información de carácter económico; ésta puede ser establecimiento único, matriz o sucursal, y fijo o semifijo” (INEGI, 2010d: 3).
} 
femenina en la ZMM; el segundo, establecer que cada uno de los municipios cumple con una función urbana específica dentro del todo metropolitano, cuestión que permitirá el planteamiento de que el espacio urbano de interés es un territorio funcionalmente zonificado 5 .

Fuente: Elaboración propia con datos del Instituto Nacional de Estadística y Geografía (INEGI, 2010f).

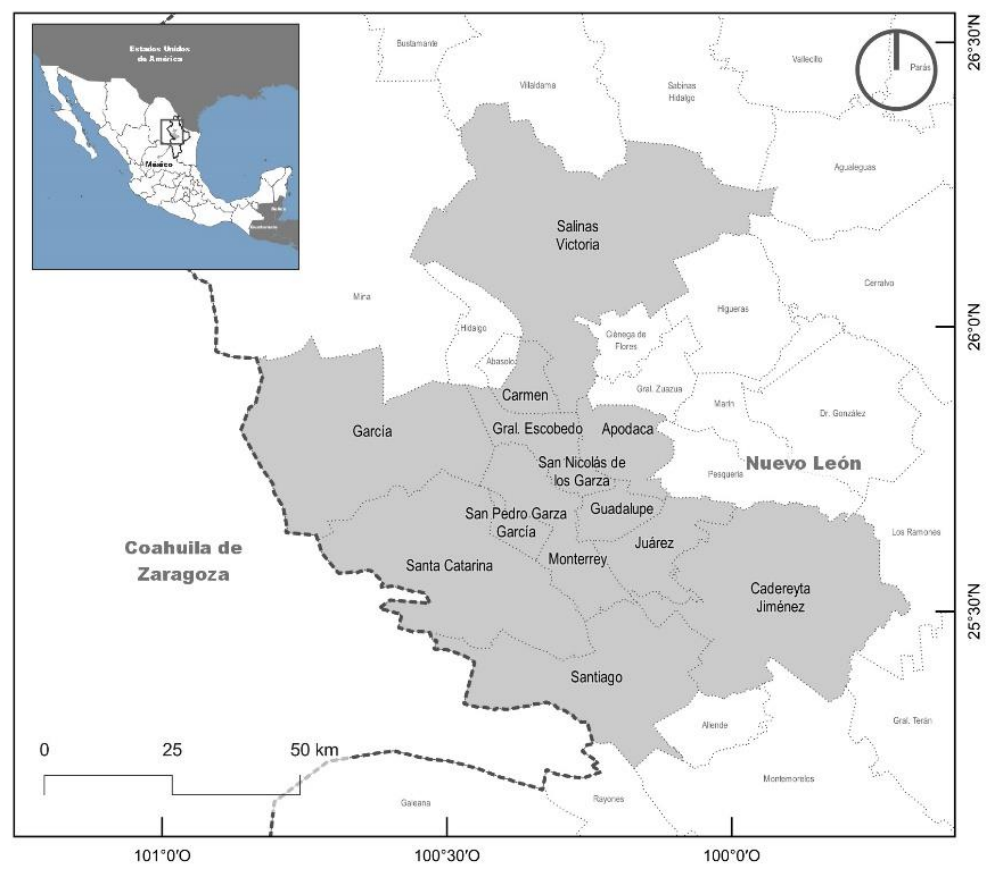

Figura 1. Localización de la zona metropolitana de Monterrey (ZMM).

Mediante la referida revisión, se logra conocer el nivel de concentración de UE por sector de actividad y por municipio, lo que permite determinar dos aspectos: 1) la distribución de UE de cada sector económico a nivel municipal en relación al total metropolitano (números absolutos); y 2) la tasa de UE por actividad económica de cada municipio con respecto al total municipal (números relativos).

Se consideran asimismo las tasas de crecimiento poblacional para el lapso de 2000-2010 (SEDESOL et al., 2012), con el propósito de identificar a los municipios de la ZMM en donde la tendencia de la población a asentarse fue más alta en esos años. En adición, se toman en cuenta los porcentajes de la población ocupada que labora en un municipio distinto al que reside en 2010, con el fin de tener una información más puntual sobre los territorios cuya participación metropolitana se vincula mayormente a la función de habitar, de modo que esta

\footnotetext{
${ }^{5}$ Los datos utilizados para tal cometido son los generados bajo la coordinación del INEGI, a través del Sistema Nacional de Información Estadística y Geográfica (SNIEG), y puestos a disposición para su consulta en el Directorio Estadístico Nacional de Unidades Económicas 2010 (DENUE) (INEGI, 2010a).
} 
se integre al estudio de la actividad económica. Ello permite conclusiones más certeras en relación a la zonificación o división funcional del espacio metropolitano.

Se establece pues que, al conjugar los análisis mencionados, la ZMM presenta la siguiente configuración funcional:

1. Centralidad urbana: dominio indudable del municipio de Monterrey, papel ahora compartido con los municipios de Guadalupe y San Nicolás de los Garza, demarcaciones situadas al centro geográfico metropolitano.

2. Zona habitacional: esta función urbana actualmente es desempeñada por los municipios de Juárez, al sureste, y de García y Escobedo, al noroeste y norte metropolitanos, respectivamente.

3. Zona industrial: aunque no se puede negar la importancia de los municipios centrales de Monterrey, Guadalupe y San Nicolás en la actividad industrial, demarcaciones como Carmen y Santa Catarina se destacan en este giro.

4. Zona financiera y de negocios: San Pedro Garza García se ha convertido en la antonomasia de la actividad terciaria regiomontana, particularmente de los servicios profesionales especializados; en él se emplaza el distrito financiero y de negocios de la ZMM.

5. Servicios educativos y de salud: el rol protagónico en la oferta de esta clase de servicios lo comparten San Pedro, San Nicolás y Monterrey.

6. Actividad comercial: en este rubro sobresale la periferia norte de la metrópoli con municipios como García, Salinas Victoria, General Escobedo, Santa Catarina y Apodaca.

7. Zona de ocio y de descanso: la periferia sur-sureste figura en las primeras posiciones de la lista de ofertantes de servicios recreativos con municipios como Santiago, Juárez y Cadereyta Jiménez.

\section{Resultados y discusión}

\section{Análisis estadístico de los datos. Regresión por mínimos cuadrados ordinarios (MCO) y regresión multi-logística}

De este par de regresiones se obtiene información valiosa acerca de la relación entre la distancia viv-TP y algunas variables demográficas (MCO) y económicas (multi-logística) de las mujeres ocupadas (MO) de la ZMM. Si bien el tamaño del universo inicial es el de las 22 408 MO de la ZMM, este se reduce a las 12468 que presentan la información completa, esto es, se eliminan las condiciones no especificado y blanco por base que aparecen en la base de datos de la Muestra censal (INEGI, 2010c) examinada. 
En la Figura 2 se puede advertir que la variable situación conyugal no es de importancia para la distancia recorrida por la MO hacia su lugar de trabajo retribuido, en tanto que su $P$-valor es de 0.9368 (mayor que 0.05). En cuanto al número de hijos, la distancia se verá reducida en $316 \mathrm{~m}$. por cada hijo que se tenga, por lo que la mujer tiende a laborar más cerca de su vivienda conforme aumenta la cantidad de estos. Por otro lado, si el nivel académico de la mujer se eleva, la distancia se incrementa también en $650 \mathrm{~m}$, a partir de lo cual se puede afirmar que, a mayor formación escolar, mayor es la separación viv-TP. Finalmente, aunque el $P$-valor de la variable ingreso mensual indique que esta es significativa para la distancia (0.00, menor que 0.05), su Coeficiente habla de un efecto muy pequeño, ya que por cada $\$ 1000.00$ más, el incremento en la distancia es de $0.059 \mathrm{~m}$. Sin embargo, la tendencia es a alejarse de la vivienda para conseguir una mejor retribución.

Fuente: Elaboración propia a partir de los datos de la Muestra censal (INEGI, 2010c).

\begin{tabular}{|lrrrc|}
\hline & Coefficients & Standard Error & $t$ Stat & P-value \\
\hline Intercept & 4113.9156 & 218.2466 & 18.8498 & 0.0000 \\
Situación Conyugal & -11.2730 & 142.1642 & -0.0793 & 0.9368 \\
Número de Hijos & -316.1478 & 46.1874 & -6.8449 & 0.0000 \\
Nivel Academico & 650.5781 & 88.6860 & 7.3357 & 0.0000 \\
Ingreso Mensual & 0.0597 & 0.0086 & 6.9288 & 0.0000 \\
\hline
\end{tabular}

Figura 2. Regresión por mínimos cuadrados ordinarios (MCO). Distancia viv-TP Vs variables demográficas

Cálculo sobre el total representativo de las ocupadas que en 2010 residían en cada municipio de la ZMM y de quienes se conoce la situación geográfica de su trabajo remunerado. No se incluyen condiciones de variables no especificadas. Ejecutado en Microsoft Excel 2013, Addins Analysis Toolpak.

Ahora bien, en lo referente a la regresión multi-logística, la magnitud de los coeficientes que esta arroja no es interpretable, de modo que la información que se presenta es la de los efectos marginales (marginal effects after mlogit en los datos de las Figuras 3 y 4). Antes de interpretar los resultados de las Figuras 3 y 4, hay que aclarar que se analizaron todos los sectores económicos en los que se ocupan las mujeres estudiadas: 1 , sector primario; 2 , sector secundario; 3, distribución de bienes (DB), actividad comercial al mayoreo y al menudeo, básicamente; 4, operaciones con información (OI); 5, operaciones con activos (OA); 6, servicios profesionales especializados (SPE); 7, servicios educativos y de salud (SES); 8, servicios recreativos (SR); 9 otros servicios (OS), principalmente servicios personales y de limpieza; y 10 , actividades gubernamentales $(\mathrm{G})^{6}$. Sin embargo, en las figuras referidas se

\footnotetext{
${ }^{6}$ La clasificación de la actividad económica utilizada por el INEGI, se basa en la establecida por el Sistema de
} Clasificación Industrial de América del Norte (INEGI, 2010e). 
han omitido el sector primario, el de las OI, OA, y el G, debido a los bajos porcentajes de participación femenina en ellos.

De acuerdo a la Figura 3, en el sector manufacturero (outcome (2)) se ubica el $22.17 \%$ de la población analizada, el segundo colectivo más grande, aunque el efecto de la variable distancia viv-TP no representa ninguna afectación sobre la probabilidad de ocuparse en él (6.93 e-07). La actividad comercial o de DB (outcome (3)) comprende al $27.38 \%$ de las mujeres ocupadas, el grupo de mayor presencia entre la población estudiada; la probabilidad de pertenecer a este disminuye un $8.96 \%$ (-8.96 e-06) por cada $10 \mathrm{~km}$, es decir, no se requiere alejarse de la vivienda para participar en esta actividad. Finalmente, solo el $7.3 \%$ de las mujeres ocupadas forman parte de los SPE (outcome (6)); al considerar la variable distancia, la tendencia de ocuparse en ellos es mayor en un $1.93 \%$ (1.93 e-06) cada $10 \mathrm{~km}$ más lejos del municipio de residencia, lo que admite declarar que la mujer que aspire a colocarse en este sector debe de alejarse considerablemente de su vivienda.

En lo que concierne a los SES (outcome (7)), la Figura 4 arroja que el 14.45\% de la población de interés está ocupada en este sector, el tercero más concurrido; el resultado indica que a cada $10 \mathrm{~km}$ de la vivienda, la probabilidad de ocuparse en él crece en un 3.32\% (3.32 e-06), por lo que la lejanía entre la residencia y el trabajo es característica de esta actividad. Se determina también que el $9.81 \%$ de las mujeres está involucrado en los SR (outcome (8)), si bien no existen razones para concluir que la variable distancia influya en la oportunidad de integrarse a este sector (2.96 e-07). Por último, en los OS (outcome (9)) se localiza el 12\% del universo de estudio; hay que señalar que es el cuarto grupo más nutrido. En cuanto a la incidencia de la distancia sobre el grado de pertenencia a este sector, llama la atención que los resultados indiquen que la probabilidad de ocuparse en él se eleve en un $2.59 \%$ (2.59 e06) por cada $10 \mathrm{~km}$ que la mujer se aparte de su vivienda.

Es de suma relevancia señalar, en principio, las grandes concentraciones de mujeres en los sectores de la DB (27.38\%), la actividad manufacturera (22.17\%), los SES (14.45\%) y los OS (12\%). Esto alude a la segregación ocupacional que prevalece entre el colectivo femenino, tradicionalmente ligado a este tipo de actividades (Hanson \& Pratt, 1995; Manning, 2017; Mcdowell, 2000; Sassen, 2002; Schwartz, s/f; Valcárcel, 2009). Por otro lado, es de sorprender el nivel de relación entre las grandes longitudes viv-TP ligadas a los SPE o los SES, los cuales se asocian a requerimientos de formación profesional o especialización, en congruencia con los resultados acerca del nivel académico de la regresión MCO. Por el contrario, la DB, poco demandante de estudios superiores, supone una situación geográfica cercana a la vivienda. Con respecto a los OS, se esperaría que representaran un centro de trabajo próximo a la vivienda, pero los resultados muestran lo contrario; la siguiente sección muestra hallazgos interesantes en relación a este sector de ocupación. 
Fuente: Elaboración propia a partir de los datos de la Muestra censal (INEGI, 2010c).

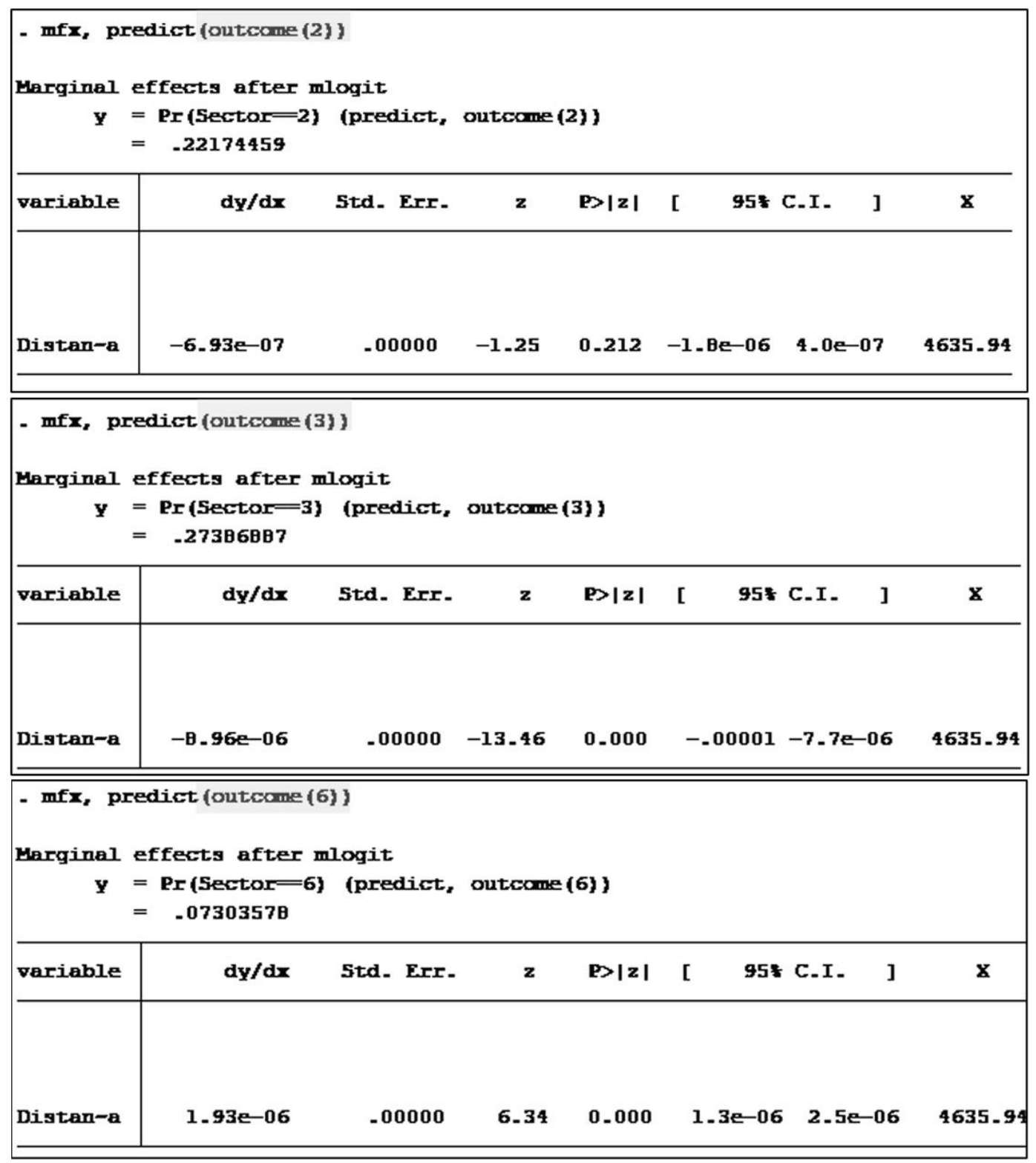

Figura 3. Regresión multi-logística ${ }^{7}$ : efectos marginales. Distancia viv-TP Vs sector económico de ocupación.

7 Cálculo sobre el total representativo de las ocupadas que en 2010 residían en algún municipio de la ZMM y de quienes se conoce la situación geográfica de su trabajo remunerado. No se incluyen condiciones de variables no especificadas. Sector secundario (2); DB (3); SPE (6). Ejecutado en Stata. 
Fuente: Elaboración propia a partir de los datos de la Muestra censal (INEGI, 2010c).

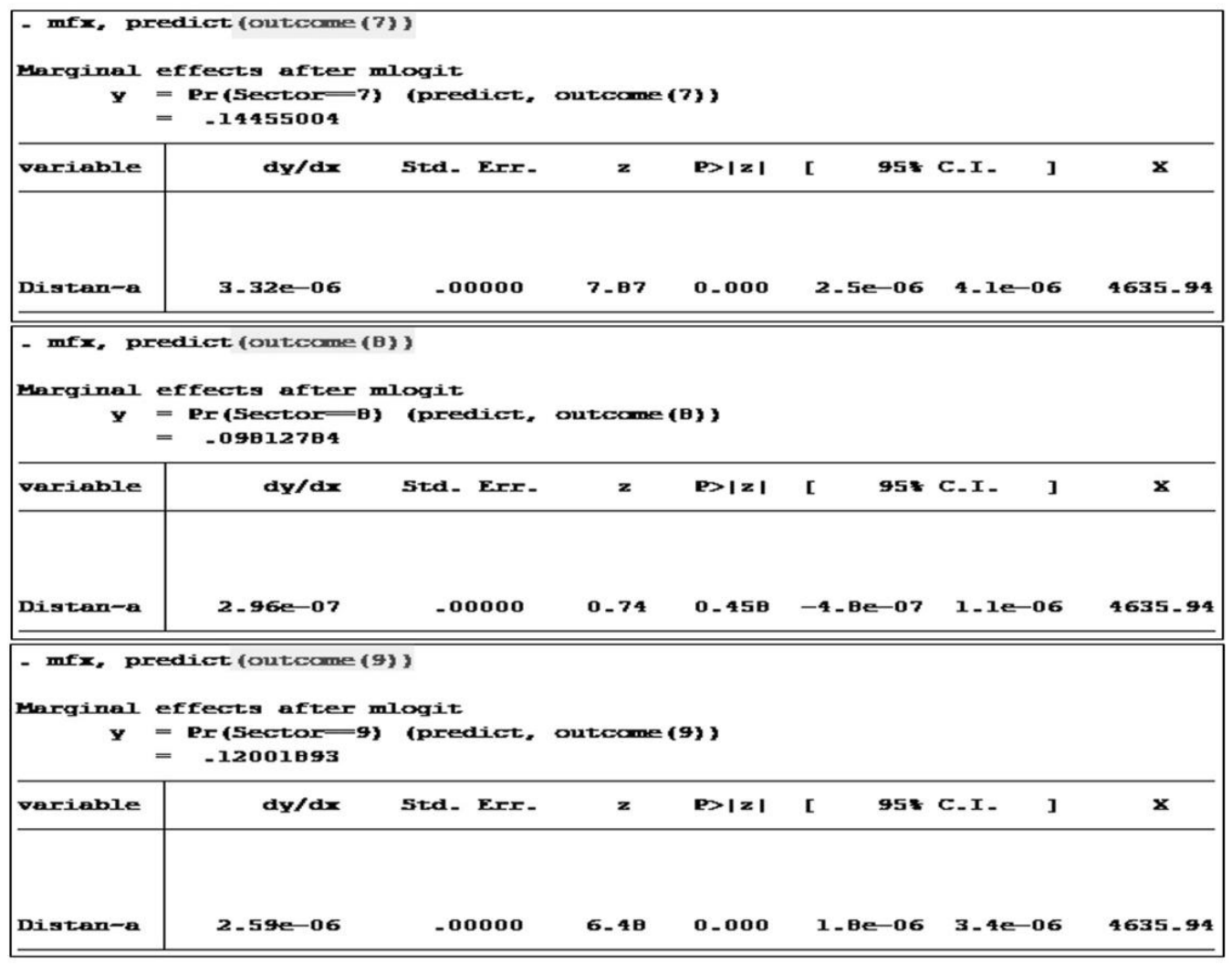

Figura 4. Regresión multi-logística ${ }^{8}$ : efectos marginales. Distancia viv-TP Vs sector económico de ocupación.

\section{Análisis comparativo de trayectos femeninos vivienda-trabajo productivo (viv-TP) de la ZMM: cargas de trabajo reproductivo (CTR)}

Con la intención de ver de manera concreta y clara lo obtenido en los análisis estadísticos precedentes, y de otorgarle la debida importancia al dato espacial, se efectúa el análisis comparativo especificado en el apartado metodológico.

En primer lugar, se vacía en un solo mapa la totalidad de los trayectos femeninos viv-TP de la Muestra censal (INEGI, 2010c), cuyos municipios residencial y laboral son distintos. De igual forma que en la sección anterior, el universo de partida es de $22408 \mathrm{MO}$; no obstante, este se acota a 8 078, ya que son evidentemente eliminadas aquellas mujeres que laboran en su municipio de residencia (13 670), así como aquellas que no reportan municipio laboral. De este vaciado se observa lo siguiente: 1) el promedio de las longitudes de los viajes viv-

\footnotetext{
8 Cálculo sobre el total representativo de las ocupadas que en 2010 residían en cada municipio de la ZMM y de quienes se conoce la situación geográfica de su trabajo remunerado. No se incluyen condiciones de variables no especificadas. SES (7); SR (8); OS (9). Ejecutado en Stata.
} 
TP calculado a escala metropolitana es de $21.77 \mathrm{~km}$.; 2) se puede decir que los trayectos vivTP más largos vistos a nivel municipal se asocian con las menores concentraciones de mujeres; en cambio, los más cortos son acompañados, en la mayoría de los casos, por los mayores porcentajes; 3) sin importar la distancia, Monterrey es el destino laboral más importante en la mayoría de los municipios metropolitanos.

A continuación, se realiza un primer filtro que elimina a todos los trayectos ubicados antes del primer cuartil, esto es, los de concentraciones menores al 25\%. El resultado es la Figura 5. En ella aparece la representación gráfica de los recorridos viv-TP acompañados por el número relativo de mujeres que los realizan, calculado desde el total municipal de las expulsadas por motivos laborales ${ }^{9}$.

Ya que tal criterio conserva trayectos con diferencias considerables tanto en sus niveles de concentración como en sus longitudes, se efectúa un segundo filtro de selección.

Fuente: Elaboración propia con datos de la Muestra censal (INEGI, 2010c).

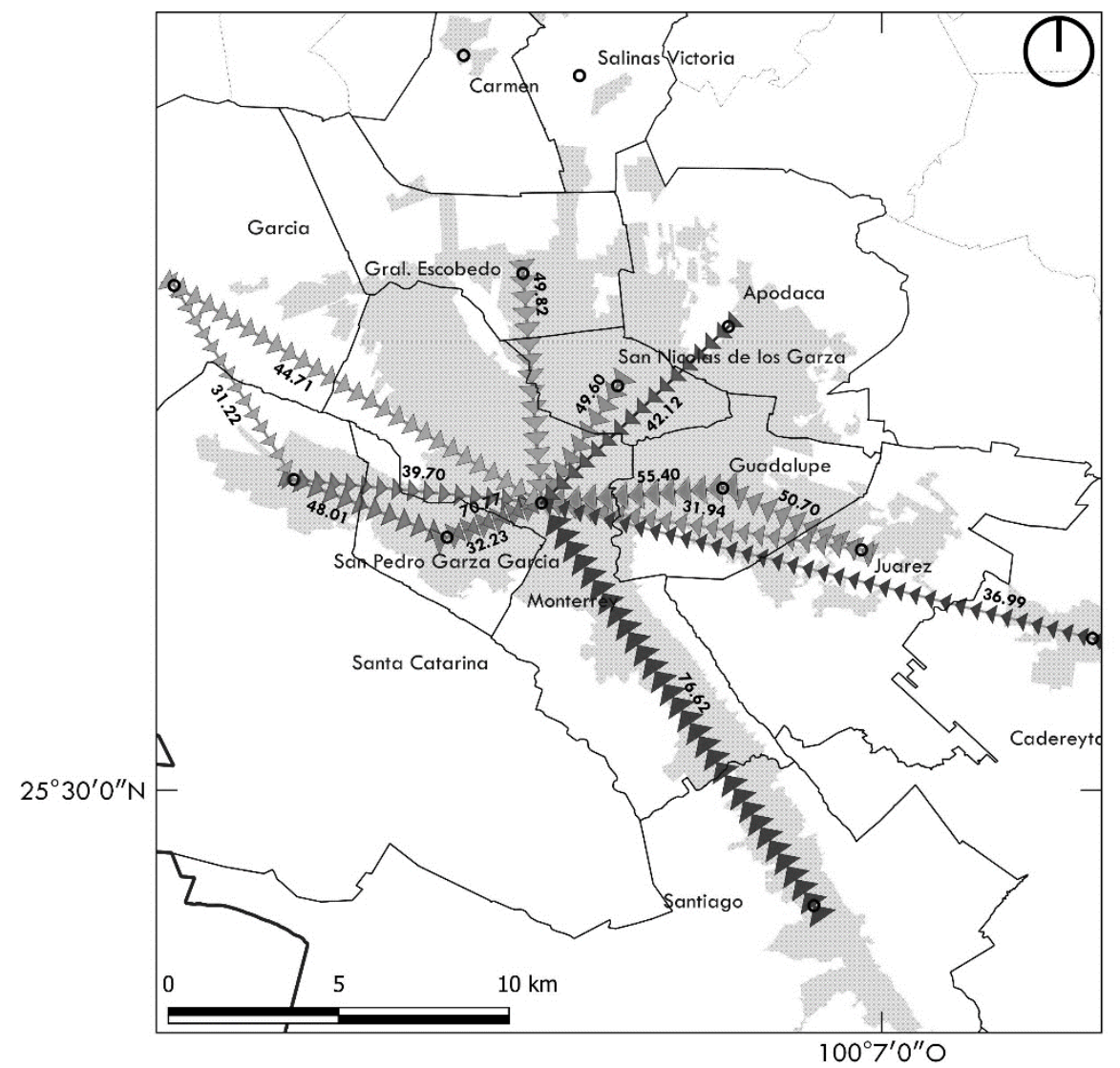

Figura 5. Trayectos viv-TP de la ZMM con concentraciones relativas de mujeres de $25 \%$ y más.

\footnotetext{
${ }^{9}$ Los trayectos cuyo destino laboral se localiza fuera de los límites de la ZMM son considerados para el cálculo del número relativo de mujeres expulsadas por motivos laborales de su municipio de residencia, mas no son representados en ninguno de los mapas aquí expuestos.
} 


\section{Trayecto de gran longitud: Cadereyta-Monterrey}

La aplicación del segundo filtro deriva en la Figura 6, el cual desaparece a los trayectos de longitudes menores al promedio de todos los viajes viv-TP, esto es, $21.77 \mathrm{~km}$. Se tiene pues a Santiago-Monterrey, de $30.39 \mathrm{~km}$ y con el $76.62 \%$ de las mujeres expulsadas de Santiago por motivos laborales; García-Monterrey, de $25.58 \mathrm{~km}$ y $44.71 \%$; y Cadereyta-Monterrey, de $33.29 \mathrm{~km}$ y $36.99 \%$. Al revisar los perfiles demográficos de las mujeres de cada uno de estos tres trayectos, el de Cadereyta-Monterrey resulta ser el de mayor interés.

La mayor parte de las mujeres de este trayecto es menor de 60 años, vive sin pareja, tiene pocos hijos y es hija del jefe o jefa del hogar, por lo que se estima que su CTR es reducida. Es deber señalar también que al interior de este grupo son más las mujeres con hijos pequeños y más también las de pocos estudios. (Tabla 2)

Fuente: Elaboración propia con datos de la Muestra censal (INEGI, 2010c).

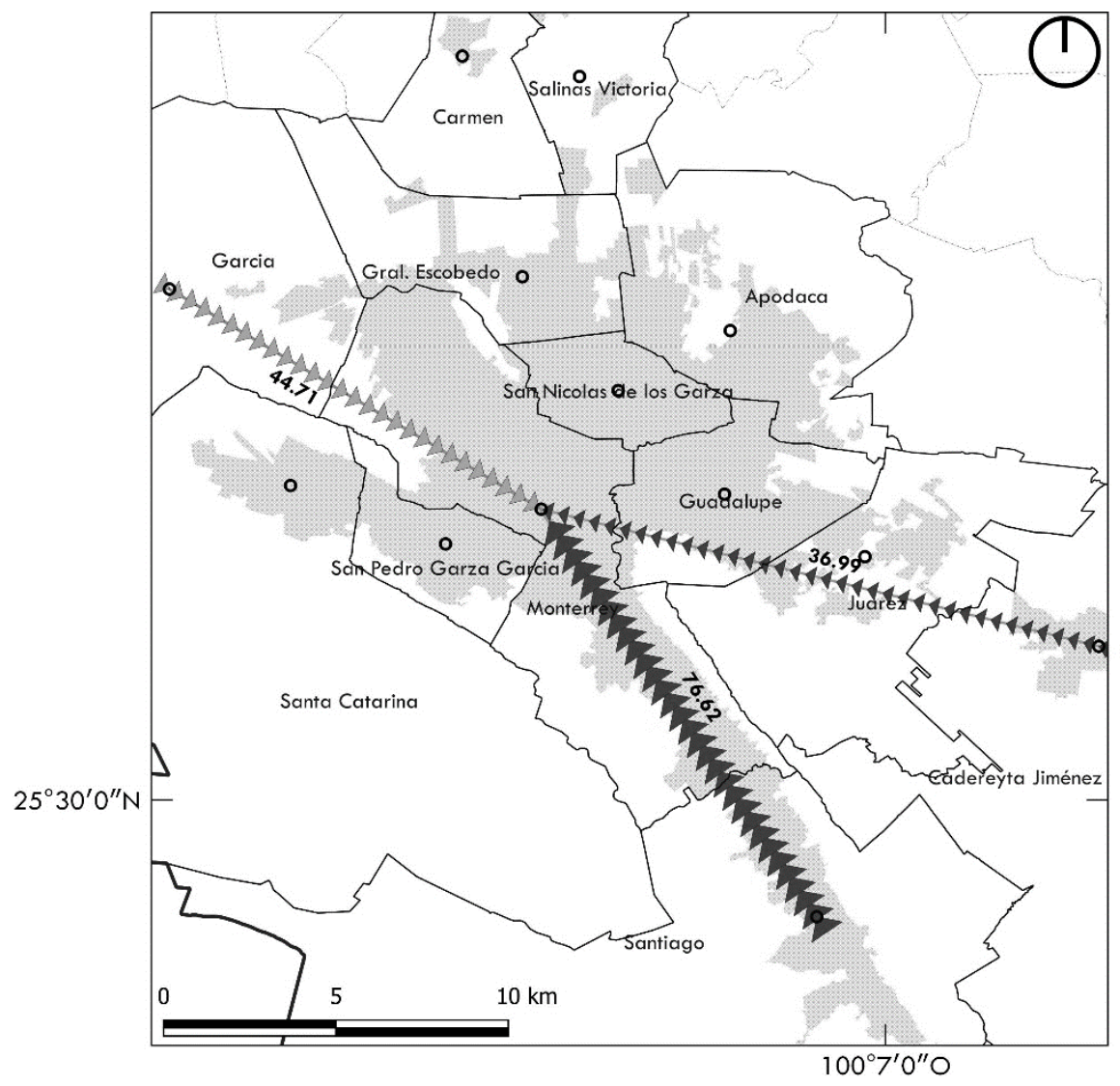

Figura 6. Trayectos viv-TP que conjugan gran distancia y alta concentración. 
Tabla 2. Porcentajes de mujeres con carga de trabajo reproductivo (CTR) reducida o considerable del trayecto de gran longitud viv-TP Cadereyta-Monterrey.

\begin{tabular}{|c|c|c|c|c|}
\hline Parentesco & Arreglo familiar & Mujeres & CTR reducida & CTR considerable \\
\hline N/A & No familiar (vive sola) & 1 & $3.70 \%$ & N/A \\
\hline Esposa & Biparental sin hijos & 0 & N/A & - \\
\hline Esposa & Biparental/hijos menores de 18 años & 9 & N/A & $33.33 \%$ \\
\hline Esposa & Biparental/hijos de 18 años y más & 0 & N/A & - \\
\hline Hija & Biparental con hijos & 5 & $18.52 \%$ & N/A \\
\hline Hija & Monoparental con hijos & 3 & $11.11 \%$ & N/A \\
\hline Jefa & Monoparental/hijos menores de 18 años & 0 & N/A & - \\
\hline Jefa & Monoparental/hijos de 18 años y más & 1 & N/A & $3.70 \%$ \\
\hline Jefa & Biparental con hijos & 1 & $3.70 \%$ & N/A \\
\hline $\begin{array}{c}\text { Esposa/Hija/ } \\
\text { Jefa/Otro }\end{array}$ & Extenso A & 5 & $18.52 \%$ & N/A \\
\hline $\begin{array}{c}\text { Esposa/Hija/ } \\
\text { Jefa/Otro }\end{array}$ & Extenso B & 2 & N/A & $7.41 \%$ \\
\hline \multicolumn{2}{|c|}{ Totales } & 27 & $56.16 \%$ & $43.84 \%$ \\
\hline
\end{tabular}

Fuente: Elaboración propia a partir de los datos de la Muestra censal (INEGI, 2010c).

La estimación de la CTR promedio se confirma tras el análisis de los arreglos familiares a los que pertenece cada una de las 27 mujeres de este trayecto. De acuerdo con la Tabla 2, el $56.16 \%$ de ellas experimenta una CTR reducida, frente al $43.84 \%$ de mujeres con CTR considerable.

\section{Trayecto de longitud nula: Cadereyta-Cadereyta}

Imprescindible es conocer la realidad del caso de las mujeres que trabajan de modo remunerado y viven en Cadereyta; es decir, aquéllas que, por algún motivo, se quedan a laborar cerca de su vivienda. Dado que la escala de los datos utilizados es la municipal, no es factible representar gráficamente el trayecto viv-TP de este grupo de mujeres, por lo que se le identifica como de longitud nula.

La mayoría de estas mujeres es menor de 60 años, vive en pareja, tiene más de un hijo y es esposa del jefe o la jefa del hogar. Esto supone una CTR mayor que la estimada para el trayecto largo Cadereyta-Monterrey. Como en ese gran trayecto, en este de longitud nula sobresale la cantidad de mujeres con hijos de 0-9 años y con una educación académica pobre.

Nuevamente esta estimación se corrobora al considerar la composición de los hogares de las sujetas estudiadas. Desafortunadamente, no es posible el estudio de la muestra entera debido a su elevado número de elementos (768), así que se acota a las mujeres que son madres de 
tres hijos o más, ya que, de entre las tres condiciones de la variable número de hijos, es la de mayor presencia.

Los datos de la Tabla 3 indican que el 83.20\%, experimenta una CTR considerable. En tanto, solo el $16.80 \%$ de quienes viven y laboran en el municipio de Cadereyta absorbe una CTR reducida.

Tabla 3. Porcentajes de mujeres con tres hijos o más, con carga de trabajo reproductivo (CTR) reducida o considerable del trayecto de longitud nula viv-TP Cadereyta-Cadereyta.

\begin{tabular}{|c|c|c|c|c|}
\hline Parentesco & Arreglo familiar & Mujeres & CTR reducida & CTR considerable \\
\hline N/A & No familiar (vive sola) & 9 & $3.44 \%$ & N/A \\
\hline Esposa & Biparental sin hijos & 19 & N/A & $7.25 \%$ \\
\hline Esposa & Biparental/hijos menores de 18 años & 97 & N/A & $37.02 \%$ \\
\hline Esposa & Biparental/hijos de 18 años y más & 26 & N/A & $9.92 \%$ \\
\hline Hija & Biparental con hijos & 0 & - & N/A \\
\hline Hija & Monoparental con hijos & 0 & - & N/A \\
\hline Jefa & Monoparental/hijos menores de 18 años & 27 & N/A & $10.31 \%$ \\
\hline Jefa & Monoparental/hijos de 18 años y más & 14 & N/A & $5.34 \%$ \\
\hline Jefa & Biparental con hijos & 1 & $0.38 \%$ & N/A \\
\hline $\begin{array}{c}\text { Esposa/Hija/ } \\
\text { Jefa/Otro }\end{array}$ & Extenso A & 34 & $12.98 \%$ & N/A \\
\hline $\begin{array}{c}\text { Esposa/Hija/ } \\
\text { Jefa/Otro }\end{array}$ & Extenso B & 35 & N/A & $13.36 \%$ \\
\hline \multicolumn{2}{|c|}{ Totales } & 262 & $16.80 \%$ & $83.20 \%$ \\
\hline
\end{tabular}

Fuente: elaboración propia a partir de los datos de la Muestra censal (INEGI, 2010c).

\section{Trayecto de poca longitud: Juárez-Guadalupe}

Con la finalidad de ofrecer datos suficientes que admitan el planteamiento de conclusiones claras mediante su cotejo, es pertinente el análisis de un segundo trayecto característicamente opuesto al de gran longitud. Se busca ahora un trayecto corto y de concurrencia alta.

Para esto, es necesario retomar la Figura 5. Bajo la consigna de retener solo a aquellos recorridos que conjuguen distancias cortas y altos porcentajes de mujeres ocupadas, se obtiene la Figura 7. Esta figura contiene los tres recorridos más cortos y de mayor concurrencia realizados por las mujeres de la ZMM por razones laborales: Juárez-Guadalupe, de $9.01 \mathrm{~km}$ y con el $50.70 \%$ de las mujeres expulsadas de Juárez a razón del trabajo remunerado; Guadalupe-Monterrey, de 10.60 km y 55.40\%; y San Pedro-Monterrey, de 5.94 $\mathrm{km}$ y $70.77 \%$. 
Fuente: Elaboración propia con datos de la Muestra censal (INEGI, 2010c).

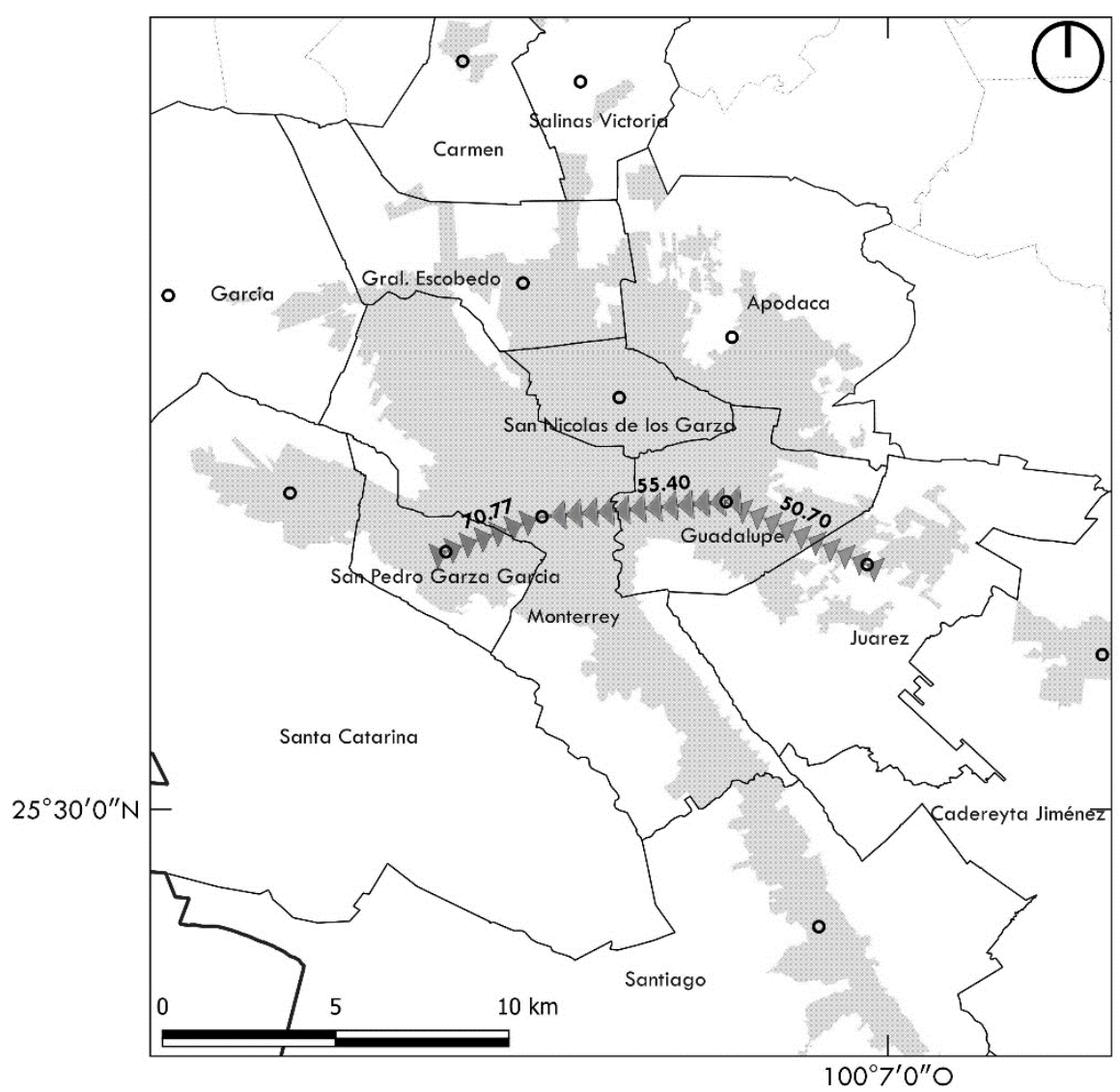

Figura 7. Trayectos vivienda-trabajo remunerado que conjugan poca distancia y alta concentración.

Como en el caso anterior, se elige a uno solo de ellos por mejor argumentar la cercanía geográfica entre la vivienda y el trabajo remunerado: Juárez-Guadalupe.

Este trayecto cuenta a una mayoría de mujeres jóvenes que viven en pareja, con dos, tres o más hijos pequeños, esposas del jefe o jefa del hogar y con un nivel de estudios bajo, perfil opuesto al del trayecto largo casi en la totalidad de las variables, asunto que lleva a suponer que la CTR es mayor para las mujeres de este recorrido corto.

El escrutinio de la composición familiar de los hogares a los que pertenecen las mujeres de este viaje, vuelve a ser congruente con tal suposición. Ya que este trayecto contiene un gran número de sujetas (581), la muestra a estudiar se limita a las mujeres que reportan tener tres o más hijos, la mayoría desde la variable de número de hijos.

La Tabla 4 da cuenta de la intensa CTR que recibe la gran mayoría de las mujeres que realizan este viaje por razones laborales: el $78.28 \%$ de ellas experimenta una CTR considerable, de cara al $21.74 \%$ con CTR reducida. 
Tabla 4. Porcentajes de mujeres con tres hijos o más, con carga de trabajo reproductivo (CTR) reducida o considerable del trayecto de poca longitud viv-TP Juárez-Guadalupe.

\begin{tabular}{|c|c|c|c|c|}
\hline \multirow{2}{*}{ Parentesco } & Arreglo familiar & Mujeres & CTR reducida & CTR considerable \\
\hline N/A & No familiar (vive sola) & 3 & $1.86 \%$ & N/A \\
\hline Esposa & Biparental sin hijos & 5 & N/A & $3.11 \%$ \\
\hline Esposa & Biparental/hijos menores de 18 años & 78 & N/A & $48.45 \%$ \\
\hline Esposa & Biparental/hijos de 18 años y más & 6 & N/A & $3.73 \%$ \\
\hline Hija & Biparental con hijos & 0 & - & N/A \\
\hline Hija & Monoparental con hijos & 0 & - & N/A \\
\hline Jefa & Monoparental/hijos menores de 18 años & 9 & N/A & $5.59 \%$ \\
\hline Jefa & Monoparental/hijos de 18 años y más & 5 & N/A & $3.11 \%$ \\
\hline Jefa & Biparental con hijos & 0 & - & N/A \\
\hline $\begin{array}{c}\text { Esposa/Hija/ } \\
\text { Jefa/Otro }\end{array}$ & Extenso A & 32 & $19.88 \%$ & N/A \\
\hline $\begin{array}{c}\text { Esposa/Hija/ } \\
\text { Jefa/Otro }\end{array}$ & Extenso B & 23 & N/A & $14.29 \%$ \\
\hline \multicolumn{2}{|c|}{ Totales } & 161 & $21.74 \%$ & $78.28 \%$ \\
\hline
\end{tabular}

Fuente: elaboración propia con datos de la Muestra censal (INEGI, 2010c).

\section{Trayecto de longitud nula: Juárez-Juárez}

Es obligado conocer lo que sucede entre las mujeres que no salen del municipio de Juárez, su residencia, para el desempeño de la actividad económica.

Las características demográficas de las mujeres de este trayecto de nula distancia JuárezJuárez describen a una mujer menor de 60 años, que vive en pareja, con dos, tres o más hijos en donde la edad del más pequeño oscila entre los 0 y los 9 años, esposa del jefe o jefa del hogar y muy poco educada, de manera similar al perfil del trayecto Cadereyta-Cadereyta, igualmente de longitud nula y para el que se valoró una alta CTR.

De manera reiterada, el examen de los arreglos familiares correspondientes constata tal valoración. Por tercera ocasión, la muestra de interés es demasiado grande como para analizarla íntegra (517 sujetas), así que esta se limita a las mujeres que declaran tener tres o más hijos, quienes constituyen la mayor parte desde la variable de número de hijos.

La Tabla 5 soporta la estimación de la alta CTR a partir de la revisión de las variables demográficas: $82.94 \%$ de las mujeres estudiadas perciben un nivel considerable de este tipo de trabajo, mientras que únicamente el $17.05 \%$ de ellas experimenta un nivel reducido.

Tabla 5. Porcentajes de mujeres con tres hijos o más, con carga de trabajo reproductivo (CTR) reducida o considerable del trayecto de longitud nula viv-TP Juárez-Juárez. 


\begin{tabular}{|c|c|c|c|c|}
\hline Parentesco & Arreglo familiar & Mujeres & CTR reducida & CTR considerable \\
\hline N/A & No familiar (vive sola) & 1 & $0.46 \%$ & N/A \\
\hline Esposa & Biparental sin hijos & 11 & N/A & $5.07 \%$ \\
\hline Esposa & Biparental/hijos menores de 18 años & 100 & N/A & $46.08 \%$ \\
\hline Esposa & Biparental/hijos de 18 años y más & 16 & N/A & $7.37 \%$ \\
\hline Hija & Biparental con hijos & 0 & - & N/A \\
\hline Hija & Monoparental con hijos & 0 & - & N/A \\
\hline Jefa & Monoparental/hijos menores de 18 años & 15 & N/A & $6.91 \%$ \\
\hline Jefa & Monoparental/hijos de 18 años y más & 4 & N/A & $1.84 \%$ \\
\hline Jefa & Biparental con hijos & 0 & - & N/A \\
\hline $\begin{array}{c}\text { Esposa/Hija/ } \\
\text { Jefa/Otro }\end{array}$ & Extenso A & 36 & $16.59 \%$ & N/A \\
\hline $\begin{array}{c}\text { Esposa/Hija/ } \\
\text { Jefa/Otro }\end{array}$ & Extenso B & 34 & N/A & $15.67 \%$ \\
\hline \multicolumn{2}{|c|}{ Totales } & 217 & $17.05 \%$ & $82.94 \%$ \\
\hline
\end{tabular}

Fuente: Elaboración propia a partir de los datos de la Muestra censal (INEGI, 2010c).

Hasta aquí, los datos muestran la clara tendencia de que a mayor CTR, menor es la distancia viv-TP. Los trayectos de nula distancia Cadereyta-Cadereyta y Juárez-Juárez ostentan porcentajes por arriba del $80 \%$ de mujeres con cargas considerables $(83.20 \%$ y $82.94 \%$, respectivamente). En tanto, el trayecto corto Juárez-Guadalupe ve relativamente disminuido el valor de las cargas considerables (78.28\%), aunque este se mantiene superior al de las cargas reducidas $(21.74 \%)$. El trayecto largo Cadereyta-Monterrey viene a consolidar dicha tendencia al exhibir valores contrarios a los recorridos mencionados: ahora es el porcentaje de mujeres con CTR reducida (56.16\%) el que prevalece por sobre el de nivel considerable $(43.84 \%)$.

\section{Análisis comparativo de trayectos femeninos vivienda-trabajo productivo (viv-TP) de la ZMM: sector económico de ocupación y nivel de ingresos}

Los datos obtenidos hasta este punto consienten la posibilidad de establecer la comparación directa entre los cuatro trayectos estudiados a partir de tres arreglos: los dos pares de viajes que representan 'ambas caras de una misma moneda', como son Cadereyta-Cadereyta (MO en su municipio de residencia) Vs Cadereyta-Monterrey (MO fuera de su municipio de residencia), y Juárez-Juárez (MO en su municipio de residencia) Vs Juárez-Guadalupe (MO fuera de su municipio de residencia); y un tercer par que enfrenta la distancia larga CadereytaMonterrey contra la corta Juárez-Guadalupe. 
Dada la importancia que el objetivo de investigación otorga a los conceptos de carga doméstica y de cuidado que experimentan las MO, distancia entre su vivienda y su trabajo remunerado, sector económico en el que se ocupan y nivel de ingresos percibido, se elaboran las Tablas 6, 7 y 8. Estas contienen la información ampliada, obtenida de la misma Muestra censal (INEGI, 2010c), perteneciente a cada uno de los pares de recorridos establecidos. Su orden de aparición responde a la disminución de la diferencia entre las distancias recorridas en un trayecto y otro de cada pareja.

En primer lugar, las diferencias entre las CTR considerables al interior de cada dupla de viajes son reveladoras, pues estas disminuyen a la par de las diferencias de la longitud recorrida. En la Tabla 6 correspondiente a Cadereyta-Cadereyta, de longitud nula (83.20\%) Vs Cadereyta-Monterrey, de 33.29 km (43.84\%), la diferencia de las CTR considerables es de 39.36 puntos porcentuales. En la Tabla 7 dedicada a Cadereyta-Monterrey, de $33.29 \mathrm{~km}$ (43.84\%) Vs Juárez-Guadalupe, de 9.01 km (78.28\%), la diferencia es de 34.44 puntos. Por último, en la Tabla 8 se aprecia a Juárez-Juárez, de longitud nula (82.94\%) Vs JuárezGuadalupe, de $9.01 \mathrm{~km}$ (78.28\%), la diferencia es de 4.66 puntos.

En lo tocante al sector económico de ocupación, si se deja de lado a la actividad comercial (DB) (ya que es una ocupación recurrente entre el sector femenino), es posible reparar en algunos aspectos relevantes.

Tabla 6. Comparativo ampliado de los trayectos viv-TP de longitud nula y largo de Cadereyta-Cadereyta y Cadereyta-Monterrey.

\begin{tabular}{|c|c|c|c|}
\hline & & Cadereyta-Cadereyta & Cadereyta-Monterrey \\
\hline \multicolumn{2}{|c|}{ Distancia viv-TP } & Nula & $33.29 \mathrm{~km}$ \\
\hline \multirow{3}{*}{ Edad } & 12-29 años & $32.68 \%$ & $44.44 \%$ \\
\hline & 30-59 años & $63.28 \%$ & $55.56 \%$ \\
\hline & 60 y más & $3.91 \%$ & - \\
\hline \multirow{2}{*}{ Situación conyugal } & En pareja & $58.20 \%$ & $40.74 \%$ \\
\hline & Sin pareja & $41.41 \%$ & $59.26 \%$ \\
\hline \multirow{3}{*}{ Número de hijos } & 1 hijo & $13.67 \%$ & $22.22 \%$ \\
\hline & 2 hijos & $23.70 \%$ & $18.52 \%$ \\
\hline & 3 o más & $34.11 \%$ & $7.41 \%$ \\
\hline \multirow{3}{*}{$\begin{array}{l}\text { Edad del hijo más } \\
\text { pequeño }\end{array}$} & $0-9$ años & $33.07 \%$ & $22.22 \%$ \\
\hline & 10-17 años & $18.23 \%$ & $18.52 \%$ \\
\hline & 18 y más & $17.58 \%$ & $3.70 \%$ \\
\hline \multirow{3}{*}{$\begin{array}{c}\text { Parentesco con el jefe } \\
\text { o jefa del hogar }\end{array}$} & Esposa & $50.65 \%$ & $37.04 \%$ \\
\hline & Hija & $27.08 \%$ & $48.15 \%$ \\
\hline & Jefa & $18.49 \%$ & $11.11 \%$ \\
\hline \multirow{2}{*}{ Nivel académico } & Sin prepa & $65.10 \%$ & $55.56 \%$ \\
\hline & Con prepa & $18.75 \%$ & $3.70 \%$ \\
\hline
\end{tabular}


Análisis de recorridos vivienda-trabajo productivo realizados por mujeres con distinta carga reproductiva en la zona metropolitana de Monterrey, México

\begin{tabular}{|c|c|c|c|}
\hline & Lic. y más & $16.02 \%$ & $40.74 \%$ \\
\hline \multirow{2}{*}{ CTR } & Reducida & $\begin{array}{l}\text { 16.80\% de las mujeres con } \\
\text { tres o más hijos }\end{array}$ & $\begin{array}{c}56.16 \% \text { del total de las } \\
\text { mujeres }\end{array}$ \\
\hline & Considerable & $\begin{array}{l}83.20 \% \text { de las mujeres con } \\
\text { tres o más hijos }\end{array}$ & $\begin{array}{c}43.84 \% \text { del total de las } \\
\text { mujeres }\end{array}$ \\
\hline \multirow{9}{*}{$\begin{array}{c}\text { Sector económico } \\
\text { de ocupación. Sobre } \\
\text { el total de las mujeres } \\
\text { de cada trayecto }\end{array}$} & $D B$ & $29.04 \%$ & $25.93 \%$ \\
\hline & $O A$ & $1.82 \%$ & - \\
\hline & $O I$ & $0.26 \%$ & - \\
\hline & $O S$ & $12.37 \%$ & $3.70 \%$ \\
\hline & Primario & $1.30 \%$ & - \\
\hline & Secundario & $21.22 \%$ & $18.52 \%$ \\
\hline & SES & $14.32 \%$ & $18.52 \%$ \\
\hline & $S P E$ & $3.39 \%$ & $14.81 \%$ \\
\hline & $S R$ & $9.51 \%$ & $11.11 \%$ \\
\hline \multirow{2}{*}{$\begin{array}{l}\text { Ingresos mensuales. } \\
\text { Sobre el total de las } \\
\text { mujeres de cada } \\
\text { trayecto }\end{array}$} & Hasta 3 SM & $57.16 \%$ & $33.33 \%$ \\
\hline & 3-12 SM & $31.55 \%$ & $51.85 \%$ \\
\hline
\end{tabular}

Fuente: Elaboración propia a partir de los datos de la Muestra censal (INEGI, 2010c).

Tabla 7. Comparativo ampliado de los trayectos viv-TP largo y corto de CadereytaMonterrey y Juárez-Guadalupe.

\begin{tabular}{|c|c|c|c|}
\hline & & Cadereyta-Monterrey & Juárez-Guadalupe \\
\hline Distancia viv & -TP & $33.29 \mathrm{~km}$ & $9.01 \mathrm{~km}$ \\
\hline & 12-29 años & $44.44 \%$ & $43.20 \%$ \\
\hline Edad & 30-59 años & $55.56 \%$ & $55.94 \%$ \\
\hline & 60 y más & - & $0.86 \%$ \\
\hline Citurán onurucl & En pareja & $40.74 \%$ & $63.34 \%$ \\
\hline Siluactom cony ugad & Sin pareja & $59.26 \%$ & $36.66 \%$ \\
\hline & 1 hijo & $22.22 \%$ & $18.93 \%$ \\
\hline Número de hijos & 2 hijos & $18.52 \%$ & $27.19 \%$ \\
\hline & 3 o más & $7.41 \%$ & $27.71 \%$ \\
\hline & $0-9$ años & $22.22 \%$ & $43.37 \%$ \\
\hline cuac der mito inas & 10-17 años & $18.52 \%$ & $18.76 \%$ \\
\hline & 18 y más & $3.70 \%$ & $10.33 \%$ \\
\hline & & Cadereyta-Monterrey & Juárez-Guadalupe \\
\hline Parentesco con el jefe & Esposa & $37.04 \%$ & $55.25 \%$ \\
\hline o jefa del hogar & Hija & $48.15 \%$ & $24.44 \%$ \\
\hline
\end{tabular}




\begin{tabular}{|c|c|c|c|}
\hline & Jefa & $11.11 \%$ & $16.18 \%$ \\
\hline \multirow{3}{*}{ Nivel académico } & Sin prepa & $55.56 \%$ & $75.56 \%$ \\
\hline & Con prepa & $3.70 \%$ & $17.38 \%$ \\
\hline & Lic. y más & $40.74 \%$ & $6.20 \%$ \\
\hline \multirow{2}{*}{ CTR } & Reducida & $\begin{array}{c}56.16 \% \text { del total de las } \\
\text { mujeres }\end{array}$ & $\begin{array}{c}21.74 \% \text { de las mujeres con } \\
\text { tres o más hijos }\end{array}$ \\
\hline & Considerable & $\begin{array}{c}43.84 \% \text { del total de las } \\
\text { mujeres }\end{array}$ & $\begin{array}{c}78.28 \% \text { de las mujeres con } \\
\text { tres o más hijos }\end{array}$ \\
\hline \multirow{9}{*}{$\begin{array}{c}\text { Sector económico } \\
\text { de ocupación. Sobre } \\
\text { el total de las mujeres } \\
\text { de cada trayecto }\end{array}$} & $D B$ & $25.93 \%$ & $18.93 \%$ \\
\hline & $O A$ & - & $1.72 \%$ \\
\hline & $O I$ & - & $0.17 \%$ \\
\hline & $O S$ & $3.70 \%$ & $11.02 \%$ \\
\hline & Primario & - & $0.34 \%$ \\
\hline & Secundario & $18.52 \%$ & $44.58 \%$ \\
\hline & SES & $18.52 \%$ & $8.43 \%$ \\
\hline & $S P E$ & $14.81 \%$ & $4.30 \%$ \\
\hline & $S R$ & $11.11 \%$ & $7.23 \%$ \\
\hline \multirow{2}{*}{$\begin{array}{c}\text { Ingresos mensuales. } \\
\text { Sobre el total de las } \\
\text { mujeres de cada } \\
\text { trayecto }\end{array}$} & Hasta 3 SM & $33.33 \%$ & $69.71 \%$ \\
\hline & 3-12 SM & $51.85 \%$ & $26.51 \%$ \\
\hline
\end{tabular}

Fuente: Elaboración propia a partir de los datos de la Muestra censal (INEGI, 2010c).

Tabla 8. Comparativo ampliado de los trayectos viv-TP de longitud nula y corto de JuárezJuárez y Juárez-Guadalupe.

\begin{tabular}{|c|c|c|c|}
\hline & & Juárez-Juárez & Juárez-Guadalupe \\
\hline Distancia $v$ & $-\mathrm{TP}$ & Nula & $9.01 \mathrm{~km}$ \\
\hline & 12-29 años & $31.14 \%$ & $43.20 \%$ \\
\hline Edad & 30-59 años & $65.57 \%$ & $55.94 \%$ \\
\hline & 60 y más & $3.29 \%$ & $0.86 \%$ \\
\hline Situción conyurol & En pareja & $73.31 \%$ & $63.34 \%$ \\
\hline Siluactont cony ugal & Sin pareja & $26.69 \%$ & $36.66 \%$ \\
\hline & 1 hijo & $12.96 \%$ & $18.93 \%$ \\
\hline Número de hijos & 2 hijos & $28.82 \%$ & $27.19 \%$ \\
\hline & 3 o más & $41.97 \%$ & $27.71 \%$ \\
\hline & 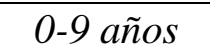 & $48.16 \%$ & $43.37 \%$ \\
\hline Edad del nijo mas & 10-17 años & $17.60 \%$ & $18.76 \%$ \\
\hline & 18 y más & $16.63 \%$ & $10.33 \%$ \\
\hline
\end{tabular}


Análisis de recorridos vivienda-trabajo productivo realizados por mujeres con distinta carga reproductiva en la zona metropolitana de Monterrey, México

\begin{tabular}{|c|c|c|c|}
\hline \multirow{3}{*}{$\begin{array}{c}\text { Parentesco con el jefe } \\
\text { o jefa del hogar }\end{array}$} & Esposa & $64.99 \%$ & $55.25 \%$ \\
\hline & Hija & $16.44 \%$ & $24.44 \%$ \\
\hline & Jefa & $14.51 \%$ & $16.18 \%$ \\
\hline \multirow{3}{*}{ Nivel académico } & Sin prepa & $80.27 \%$ & $75.56 \%$ \\
\hline & Con prepa & $12.96 \%$ & $17.38 \%$ \\
\hline & Lic. y más & $5.61 \%$ & $6.20 \%$ \\
\hline \multirow{2}{*}{ CTR } & Reducida & $\begin{array}{c}17.05 \% \text { de las mujeres con } \\
\text { tres o más hijos }\end{array}$ & $\begin{array}{c}21.74 \% \text { de las mujeres con } \\
\text { tres o más hijos }\end{array}$ \\
\hline & Considerable & $\begin{array}{l}82.94 \% \text { de las mujeres con } \\
\text { tres o más hijos }\end{array}$ & $\begin{array}{c}78.28 \% \text { de las mujeres con } \\
\text { tres o más hijos }\end{array}$ \\
\hline \multirow{9}{*}{$\begin{array}{c}\text { Sector económico } \\
\text { de ocupación. Sobre } \\
\text { el total de las mujeres } \\
\text { de cada trayecto }\end{array}$} & $D B$ & $50.29 \%$ & $18.93 \%$ \\
\hline & $O A$ & $1.74 \%$ & $1.72 \%$ \\
\hline & $O I$ & $0.58 \%$ & $0.17 \%$ \\
\hline & $O S$ & $9.28 \%$ & $11.02 \%$ \\
\hline & Primario & $0.58 \%$ & $0.34 \%$ \\
\hline & Secundario & $12.38 \%$ & $44.58 \%$ \\
\hline & $S E S$ & $5.03 \%$ & $8.43 \%$ \\
\hline & $S P E$ & $3.09 \%$ & $4.30 \%$ \\
\hline & $S R$ & $14.12 \%$ & $7.23 \%$ \\
\hline \multirow{2}{*}{$\begin{array}{l}\text { Ingresos mensuales. } \\
\text { Sobre el total de las } \\
\text { mujeres de cada } \\
\text { trayecto }\end{array}$} & Hasta 3 SM & $73.70 \%$ & $69.71 \%$ \\
\hline & 3-12 SM & $20.30 \%$ & $26.51 \%$ \\
\hline
\end{tabular}

Fuente: Elaboración propia a partir de los datos de la Muestra censal (INEGI, 2010c).

En el sector manufacturero, si bien la concentración relativa de mujeres es mayor en los viajes de menor longitud de las primeras dos parejas (Cadereyta-Cadereyta con 21.22\%, frente al $18.52 \%$ de Cadereyta-Monterrey; Juárez-Guadalupe con $44.58 \%$ frente a CadereytaMonterrey con $18.52 \%$ ), en la tercera pareja esta concentración es sumamente mayor en el trayecto más largo (Juárez-Guadalupe con $44.58 \%$, el sector con mayor ocupación relativa de ese viaje, frente a Juárez-Juárez con 12.38\%). Quizá este sea un indicio de que, en lo que al giro ocupacional respecta, está en juego no solo la distancia viv-TP, sino también el perfil económico del destino laboral. Guadalupe, considerado centralidad urbana en esta investigación, observa una posición importante en el nivel de aglutinación de UE manufactureras. Por su parte, Juárez destaca por sus servicios recreativos (SR), los que se sitúan en segundo lugar entre las ocupaciones femeninas, después de la DB y por encima del sector secundario, en el tercer puesto. 
Otra circunstancia es la que se da con relación a actividades mejor pagadas como los SPE y los SES, en los que se observan menores cantidades relativas de mujeres en la totalidad de los trayectos más cortos, si se les compara con trayectos de distancias mayores. En las tres parejas de viajes, los mayores porcentajes los poseen los recorridos de mayor longitud como Cadereyta-Monterrey (en la primera y segunda pareja, Tablas 6 y 7); y Juárez-Guadalupe (en la tercera pareja, Tabla 8). Aquí se cumple: a mayor longitud viv-TP, ocupación que implica mayor nivel académico.

Un dato más de la mayor pertinencia es el relacionado con los OS. El asunto de que, a menor longitud, ocupación que requiere menor nivel académico, se cumple solo en las primeras dos parejas de viajes. La tercer pareja, Juárez-Juárez Vs Juárez-Guadalupe, esto no se observa, pues el porcentaje de mujeres que laboran en los OS es mayor en el segundo caso.

Para concluir, las observaciones sobre el nivel de ingresos de cada pareja de recorridos son muy elocuentes. En todos los casos, hay más mujeres percibiendo ingresos superiores en desplazamientos que implican mayores distancias, en contraste con el menor número relativo de féminas percibiendo ingresos menores. Y a la inversa, hay menos mujeres con ingresos superiores en trayectos cortos, en relación al mayor número de quienes perciben menos. Estas diferencias se acortan en el par de viajes cuya discrepancia de longitudes es la menor: JuárezJuárez Vs Juárez-Guadalupe, Tabla 8.

\section{CONCLUSIONES}

Los resultados obtenidos tras los análisis estadístico y espacial llevados a cabo insinúan algún tipo de conexión entre la intensidad de la labor reproductiva y la localización, en función de su vivienda, del trabajo remunerado de la mujer.

Apuntan que, a mayor CTR, menor es la distancia viv-TP de las mujeres de la ZMM, y viceversa.

Refieren también que los sectores de ocupación de las mujeres guardan cierto grado de relación con respecto a la longitud de los trayectos femeninos viv-TP de la ZMM. Más mujeres se están ubicando en trabajos con mejor percepción social y mayor demanda de especialización profesional en los trayectos más largos, como los servicios educativos y de salud (SES) y los profesionales especializados (SPE). En cambio, se encuentra que hay más mujeres en actividades menos reconocidas en los trayectos cortos, como la actividad manufacturera o los servicios personales y de limpieza del sector de los otros servicios (OS).

Esta circunstancia se manifiesta en el nivel de ingresos de las mujeres de la ZMM: perciben más quienes más se alejan de su vivienda, y menos quienes laboran en sus proximidades.

Por lo anterior, es posible declarar que la hipótesis de partida ha sido confirmada; sin embargo, esta debe ser matizada. No solo la longitud de los desplazamientos determina el tipo de ocupación, ya que es posible identificar un factor más relacionado al giro ocupacional 
de la mujer de la ZMM: el perfil o vocación del municipio laboral. Independientemente de que el traslado sea relativamente largo, ese viaje puede derivar en ocupaciones como la manufacturera, ligada a niveles bajos de especialización y remuneración, lo que probablemente esté conectado a la actividad económica predominante del destino laboral.

Esta investigación reconoce también que los viajes seleccionados para el estudio corren en dirección periferia-centro urbano, razón por la que no es de sorprender el incremento tanto en la especialización profesional como en la percepción económica de las ocupaciones en destinos laborales como Guadalupe o Monterrey, identificados aquí como centralidad urbana. Esto es lo esperado para territorios con economías centralizadas dentro de configuraciones espaciales funcionalmente fragmentadas, como la ZMM.

En torno a esto último, se argumenta lo siguiente: 1) los desplazamientos que van de los municipios centrales hacia la periferia, no son importantes dado el reducido número relativo de mujeres que los realizan, motivo por el que no fueron considerados en el estudio; y 2) el asunto más relevante para esta disertación es enfatizar que son las mujeres con cargas de trabajo doméstico y de cuidado considerables quienes tienen la menor posibilidad de alejarse lo suficiente de su vivienda con el objetivo de aproximarse a los municipios centrales, en busca de las mejores oportunidades laborales.

Si se atiende en particular a cada uno de los tres pares de viajes analizados en la fase espacial, Cadereyta-Monterrey frente a Cadereyta-Cadereyta, Cadereyta-Monterrey ante JuárezGuadalupe, y Juárez-Guadalupe contra Juárez-Juárez, los resultados son consistentes para todos ellos: las mujeres con mayores cargas reproductivas se separan menos de su vivienda que las mujeres que experimentan menores cargas. Esas mujeres se ubican en actividades que requieren menor especialización profesional y perciben menores ingresos.

Por lo tanto, es válido suponer que las mujeres de la ZMM que tienden a distanciarse físicamente poco de su vivienda a razón del trabajo remunerado son las de 60 años y más; las que viven en pareja; las que tienen dos, tres o más hijos; las que tienen hijos pequeños; las que son esposas o jefas de hogar; y las que presentan pocos estudios.

En consecuencia, son estas mujeres, cuyas características demográficas propician CTR considerables, quienes potencialmente están siendo segregadas en el espacio urbano, en el ámbito de la ocupación productiva y, por último, en el de la percepción económica.

La principal reflexión que deja esta discusión es que la división funcional del territorio, conectada de manera importante con la actividad económica de cada municipio para el caso de la ZMM, hace pensar en una distribución espacial heterogénea de la oferta laboral que afecta grandemente a las mujeres con alta responsabilidad en la actividad doméstica y de cuidado. Es admisible estimar que este hecho se agrava en el caso de quienes residen en la periferia metropolitana.

De cara a esta circunstancia, es preciso ser pragmáticos y decir que mientras persista la baja participación del varón en las tareas no retribuidas (Alcañiz, 2015; Durán, 2005; Hanson \& 
Pratt, 1995; Mojica, 2014; OIT, 2016, 2018; Orozco, 2017; Radl, 2010), y la configuración de nuestras ciudades y de nuestra sociedad siga propiciando la separación de los ámbitos público y privado, seguirá siendo la mujer, como históricamente lo ha sido, la principal encargada de tales tareas, tema que debe ser cuestionado, también, desde la perspectiva geográfica.

Para finalizar, se considera importante la realización de trabajos futuros que contemplen la aproximación cualitativa, pues la experiencia subjetiva de las mujeres de interés ayudaría a comprender de manera más amplia la problemática urbana y social aquí abordada. Dicha aproximación permitiría el uso de herramientas analíticas como la geografía del tiempo, la cual enfatizaría las implicaciones cotidianas de la división sexual del trabajo, en la medida en que se conocería a detalle el uso del espacio-tiempo y las dificultades experimentadas principalmente por las mujeres en la organización de la vida diaria (Scholten et al., 2012).

\section{REFERENCIAS BIBLIOGRÁFICAS}

Alcañiz, M. (2015). Género con clase: la conciliación desigual de la vida laboral y familiar. RES. Revista Española de Sociología, (23), 29-55.

Aparicio Moreno, C. E., Ortega Rubí, M. E., \& Sandoval Hernández, E. (2011). La segregación socio-espacial en Monterrey a lo largo de su proceso de metropolización. Región y sociedad, 23(52), 173-207.

Belaunzarán Chavez, L. G. (2017). Geografía de género: desigualdad económica, laboral, social y commuting en la zona metropolitana del Valle de México, 2010. Universidad Autónoma del Estado de México.

Col·lectiu Punt 6. (2019). Urbanismo feminista. Por una transformación radical de los espacios de vida. Virus.

Contreras, C. (2007). Geografía de Nuevo León. Fondo Editorial de Nuevo León.

Darke, J. (1998). La ciudad modelada por el varón. En C. Booth, J. Darke, \& S. Yeandle (Eds.), La vida de las mujeres en las ciudades. La ciudad, un espacio para el cambio (pp. 115-130). Narcea.

Durán, M. (2005). Mujeres y hombres en el siglo XXI. Cuenta y razón, 138, 45-60.

Fagnani, J., \& Chauviré, Y. (1989). La actividad profesional de las mujeres con hijos en la aglomeración parisina (1975-1982). Documents d'Analisi Geografica. (15), 39-65. 
García, C. (2016). Teorías e historia de la ciudad contemporánea. Editorial Gustavo Gili, SL.

Garza, G. (2010). La transformación urbana de México, 1970-2020. En G. Garza \& M. Schteingart (Eds.), Los grandes problemas de México II. Desarrollo urbano y regional (pp. 31-86). El Colegio de México.

Greene, W. H. (2008). Econometric analysis. Prentice-Hall.

Hanson, S., \& Pratt, G. (1991). Job Search and the Occupational Segregation of Women. Annals of the Association of American Geographers, 81(2), 229-253. https://doi.org/10.1111/j.1467-8306.1991.tb01688.x

Hanson, S., \& Pratt, G. (1995). Gender, work and space. Routledge.

Instituto Nacional de Estadistica y Geografía. INEGI. (2010a). Directorio estadístico nacional de unidades económicas. Entidad: Nuevo León. 2010. DENUE. https://bit.ly/301DLCQ

Instituto Nacional de Estadistica y Geografía. INEGI. (2010b). Diseño de la muestra censal 2010. Censo de Población y Vivienda 2010. https://bit.ly/3dttQcp

Instituto Nacional de Estadistica y Geografía. INEGI. (2010c). Muestra (cuestionario ampliado). Censo de Población y Vivienda 2010. https://bit.ly/33diGqx

Instituto Nacional de Estadistica y Geografía. INEGI. (2010d). Norma técnica para la incorporación y actualización de información al Directorio estadístico nacional de unidades económicas. SNIEG. Normatividad histórica. https://bit.ly/31FK1Ig

Instituto Nacional de Estadistica y Geografía. INEGI. (2010e). Síntesis metodológica del sistema de clasificación industrial de América del Norte, México. SCIAN 2007.

Instituto Nacional de Estadistica y Geografía. INEGI. (2010f). Sistema para la consulta de información censal (SCINCE Versión 05/2012). https://bit.ly/3DvHeqT

Instituto Nacional de Estadistica y Geografía. INEGI. (2011). Principales resultados del censo de población y vivienda 2010. Nuevo León.

Madden, J. (1981). Why women work closer to home. Urban Studies, 18 (2), 181-194. https://doi.org/10.1080/00420988120080341 
Manning, A. (2017). The gender pay gap. The Economist. https://econ.st/3IvGZA4

Massey, D. (1994). Space, place, and gender. University of Minnesota Press.

Mcdowell, L. (2000). Género, identidad y lugar. Un estudio de las geografías feministas. Ediciones Cátedra.

Mojica, C. (2014). Hábitat urbano y vida cotidiana: una mirada de género a la organización espacio-temporal de las actividades en León, México. Universidad Complutense de Madrid.

Næss, P. (2006). Urban Structure Matters. Residential Location, Car Dependence and Travel Behaviour. Routledge.

Organización Internacional del Trabajo. OIT. (2016). Las mujeres en el trabajo: tendencias de 2016.

Organización Internacional del Trabajo. OIT. (2018). Perspectivas sociales y del empleo en el mundo: avance global sobre las tendencias del empleo femenino 2018.

Orozco, K. (2017). Reparto del trabajo doméstico y extradoméstico al interior de los arreglos familiares urbanos. En J. Nájera, B. García, \& E. Pacheco (Eds.), Hogares y trabajadores en México en el siglo XXI (pp. 277-326). El Colegio de México. https://doi.org/10.2307/j.ctv4v321q.9

Radl, R. M. (2010). Diferencias de género, empleo de las mujeres y el nuevo rol de género femenino. En R. M. Radl (Ed.), Investigaciones actuales de las mujeres y del género (Vol. 4, pp. 91-107). Universidad de Santiago de Compostela.

Salazar, C. E. (1999). Espacio y vida cotidiana en la ciudad de México. El Colegio de México._https://doi.org/10.2307/j.ctv3f8qgp.1

Sassen, S. (2002). Global cities and survival circuits. En B. Ehrenreich \& A. Russell (Eds.), Global woman. Nannies, maids, and sex workers in the economy (pp. 254-317). Henry Holt and Company.

Scholten, C., Friberg, T., \& Sandén, A. (2012). Re-reading time-geography from a gender perspective: examples from gendered mobility. Tijdschrift voor Economische en Sociale Geografie, 103(5), 584-600. https://doi.org/10.1111/j.1467-9663.2012.00717.x 
Análisis de recorridos vivienda-trabajo productivo realizados por mujeres con distinta carga reproductiva en la zona metropolitana de Monterrey, México

Schwartz, R. (s/f). La "revolución industrial" en el hogar: tecnología doméstica y cambio social en el siglo XX. En C. Carrasco, C. Borderías, \& T. Torns (Eds.), El trabajo de cuidados. Historia, teoría y políticas (pp. 97-121). Catarata.

Secretaría de Desarrollo Social., Consejo Nacional de Población., y Instituto Nacional de Estadística y Geografía. (2012). Delimitación de las zonas metropolitanas de México 2010 .

Segovia, O., \& Rico, M. N. (2017). ¿Cómo vivimos la ciudad? Hacia un nuevo paradigma urbano para la igualdad de género. En M. N. Rico \& O. Segovia (Eds.), ¿Quién cuida en la ciudad? Aportes para políticas urbanas de igualdad (pp. 41-70). CEPAL. https://doi.org/10.18356/f1f45bd8-es

Sobrino, J. (2003). Zonas metropolitanas de México en 2000: conformación territorial y movilidad de la población ocupada. Estudios Demográficos y Urbanos, 18(3), 461-507. https://doi.org/10.24201/edu.v18i3.1156

Valcárcel, A. (2009). Feminismo en el mundo global. Cátedra.

Vásquez, B. I. (2009). Una visión mítica de estado industrial: la hegemonía del sector terciario en las ciudades de Nuevo León, 1980-2003. En G. Garza \& J. Sobrino (Eds.), Evolución del sector servicios en ciudades y regiones de México (pp. 479-549). El Colegio de México.

Wooldridge, J. M. (2015). Introducción a la econometría. CENGAGE Learning. 